\title{
Towards Protecting Privacy in Retrieval of Image
}

\author{
Devi. T, N. Suhbakaran
}

\begin{abstract}
Protection assurance in Content Based Image Retrieval (CBIR) is another exploration point in digital security and security. The state of-workmanship CBIR structures generally get wise instrument, specifically criticalness analysis, to improve the recuperation precision. Directions to guarantee the customer's assurance in such Relevance Feedback based CBIR $(\mathrm{RF}-\mathrm{CBIR})$ is a test issue. In this paper, we investigate this issue and propose another Private Relevance Feedback CBIR (PRFCBIR) scheme. PRF-CBIR can utilize the execution increment of congruity info and spare the customer's request point meanwhile. The new PRF-CBIR involves three stages: 1) private inquiry; 2) private information; 3) neighbourhood recuperation. Private request plays out the fundamental inquiry with a security controllable component vector; private input develops the criticism picture set by presenting confounding classes following the K-secrecy standard; nearby recovery at long last re-positions the pictures in the client side. Security investigation demonstrates that PRF-CBIR satisfies the protection necessities. The analyses completed on this present reality picture gathering affirm the viability of the proposed PRF-CBIR plot.
\end{abstract}

Keyword: Content Based Image Retrieval (CBIR), PRF-CBIR

\section{INTRODUCTION}

In today's world visual data needs to be responsible for perhaps greatest idea of overall Internet traffic in both corporate and individual use circumstances [1]. The proportion of pictures, delineations, and photos being delivered and shared standard is creating at a reliably growing rate. Limit prerequisites for such a great deal of data is the driving component for data redistributing organizations, for instance, the ones using Cloud Storage as well as Computing plans. Such organizations (for instance Instagram as well as Flickr) was represented to be one of the greatest creating web organizations [2]. Furthermore, without trying to hide and private stores ordinarily prompts the necessity for substance based chase and recuperation courses of action (CBIR) [3]. Notwithstanding the manner in which that data redistributing (for example turning to distributed computing frameworks) shows up a trademark answer for reinforce generous scale picture storing and recuperation systems, it truly brings new troubles up with respect to data control including security. So, the result of redistributing data, that ordinarily induces releasing control (a couple of times full ownership) above it [4]. Continuous news was given clear confirmations so that security will not be depended upon to be shielded from providers [5], [6].

\section{Revised Manuscript Received on July 10, 2019}

Devi.T, Assistant Professor, Saveetha School of Engineering, Saveetha Institute of Medical and Technical Sciences, Thandalam, Chennai, Tamilnadu, India- 602105

N.Suhbakaran, UG Scholar, Saveetha School of Engineering, Saveetha Institute of Medical and Technical Sciences, Thandalam, Chennai, Tamilnadu, India- 602105
Additionally, malignant structure heads working for providers have complete access over data on encouraging cloud machines [7], [8]. At last, outside software engineers know how to manhandle programming vulnerabilities to build unapproved access the servers [9]. Continuous scene with iCloud picture amassing organization as well as big name photograph spillage [10] shows the significance of dangers intended to cloud-based visual information stores. Ordinary way to deal with location protection in this setting is to encode delicate information before re-appropriating it what's more, run all calculations on the customer side [11]. Be that as it may, this forces an excess of customer overhead, as information must constantly be downloaded, decoded, prepared and safely re-transferred. Numerous applications can't adapt to this overhead, especially on the web and versatile applications working over extensive datasets, for example, picture storehouses with CBIR administrations. An increasingly reasonable methodology is redistribute calculations and performs tasks over.

\section{LITERATURE SURVEY}

Image search has been broadly sent in numerous applications for the rich substance that pictures contain. In the time of enormous information, picture web crawlers must be facilitated in server farms. As a feasible arrangement, redistributing the picture search to open mists is a monetary decision for some little associations. Be that as it may, the same number of pictures contains delicate data, e.g., social insurance data and individual faces/areas, straightforwardly redistributing picture search administrations to open mists clearly raises security concerns. With this perception, a few endeavors are made towards secure picture search over scrambled dataset, yet they are constrained by either search exactness or search productivity. In this paper, we propose a lightweight secure picture search conspire over scrambled information, to be specific SEISA. Contrasted and picture search systems over plaintexts, SEISA just increments about $9 \%$ search expense and forfeits about 3\% on pursuit exactness. SEISA additionally productively supports search access control by utilizing a novel polynomial based plan, which empowers information proprietors to characterize who can look through a particular picture. Moreover, we structure a protected k-implies re-appropriating calculation that essentially spares the information proprietor's expense. To exhibit SEISA's presentation, we execute a model of SEISA on Amazon EC2 cover over a dataset with 10 million images[1]. 
Quite a bit of past consideration on choice trees centres around the part criteria and improvement of tree sizes. The difficulty between over fitting and accomplishing most extreme exactness is only here and there settled. A strategy to develop a choice tree based classifier is suggested that keeps up most elevated precision on preparing information and enhances speculation exactness as it develops in multifaceted nature. The classifier comprises of different trees developed efficiently by pseudo haphazardly choosing subsets of segments of the component vector, that is, trees built in arbitrarily picked subspaces. The subspace technique is contrasted with single-tree classifiers and other woodland development strategies by analyses on freely accessible datasets, where the strategy's predominance is illustrated. We additionally talk about freedom between trees in a woodland and relate that to the consolidated grouping accuracy.[2]

Numerous zones of science rely upon exploratory information examination and representation. The need to dissect a lot of multivariate information raises the principal issue of dimensionality decrease: how to find minimal portrayals of high-dimensional information. Here, we present locally direct installing (LLE), a solo learning calculation which figures low-dimensional, neighbourhoodprotecting implanting of high-dimensional information sources. In contrast to bunching methods that neighbourhood dimensionality decrease, LLE connects the contributions to a private international arrangement of lower dimensionality, and its improvements don't include nearby minima. By misusing neighbourhood symmetries of direct recreations, LLE can get familiar with worldwide organization of nonlinear manifolds, consider, those produced beside pictures of appearances or else archives of text.

We break down the idea of the significance criticism issue in a persistent portrayal space with regards to sight and sound data recovery. Accentuation is put on investigating the uniqueness of the issue and looking at the presumptions, executions, and benefits of different arrangements in the writing. An endeavor is made to gather a rundown of basic issues to think about when planning an importance criticism calculation. With a far reaching audit as the fundamental segment, this paper likewise offers some novel arrangements and points of view all through the discussion.[4]

A client masterminded instrument for CBIR methodology in context on a characteristic hereditary estimation (IGA) is proposed. The eventual outcomes of the proposed methodology have exhibited the basic change in recuperation execution. Also, work considering even more low-level picture descriptors or anomalous state semantics in the proposed methodology is in advance.[5]

Shown a source and channel coding structure for data stowing without end, allowing any trade-off between the detectable quality of bending displayed, the proportion of data introduced, and the dimension of capacity to fuss. The present work isn't vivacious to changes, for instance, upset and area. Changes or features of an image that are invariant to such changes should be analyzed to review this burden[6]

For transductive picture gathering, a flexible hypergraph learning system is proposed. In our method, we produce hyperedges by interfacing pictures and their nearest neighbors. This technique at the same time takes in the names of unlabeled pictures and the loads of hyperedges. The methodology investigates a solid hyperedge advancement system as well as presents a synchronous learning of the names of unlabeled pictures and the loads of hyperedges. Afterward on, they will precisely consider the philosophies and develop new frameworks to make the adaptable hypergraph learning compelling for extensive scale databases.[7]

Proposed three profitable features are shown: security against fragmentary watermark departure, security in watermark check and non-revocation. The test occurs exhibit that watermarking question pictures don't impact the recuperation execution; so the intensity of modernized watermark can be improved by extending its quality. There is no adjustment in the quality of cutting edge watermarks. As a future work, becoming new watermarking and recuperation estimations to improve the quality of electronic watermark at the expense of low quality watermarked question picture without affecting the recuperation execution[8]

Proposed a general transductive learning structure named summed up complex positioning based picture recovery (gMRBIR) for picture recovery. Exploratory outcomes on a universally useful picture database demonstrate that in the initial recovery organize, gMRBIR is vastly improved than conventional strategies in view of pairwise separations; in importance input, gMRBIR reliably enhances the recovery result and beats SVM.[9]

Shown a preliminary appraisal of interest zone descriptors inside seeing veritable geometric and photometric changes. In most of the tests, GLOH procures the best results, about took after by SIFT. This shows the power and the specific character of the region based SIFT descriptor. Shape setting moreover shows an unrivalled. The examination showed here isn't thorough and it is captivating to consolidate more scene orders. An evaluation of the descriptors concerning surface request and gathering of similar neighbourhood structures will be a useful and critical development of our work.[10]

\section{PROPOSED SYSTEM}

Watermarking in Customer Side embedding systems have proposed as possible response for copyright protection in generous substance of the scale scattering conditions. The proposed approach licenses to successfully solidify the security of client side introduce ding with the quality of instructed embedding strategies. Since this methodology licenses to successfully solidify the safe embedding of fingerprints at the client concur with the overwhelming generosity of taught introducing frameworks, giving another serious mechanical assembly to the ensured scattering of splendid intuitive media sub-position. In any case, the security isn't overhauled and when the server appropriates encoded picture it can't be in a perfect world com-pressed.

Open issues in the proposed structure to be kept an eye on later on ask about concern the prerequisite for higher security furthermore, 
the weight overhead. Especially masterminded guidelines to remain the customer's confirmation as in Relevance Feedback based CBIR (RF-CBIR) that stands as a test issue. The proposed system look at this issue and propose another Private Relevance Feedback CBIR (PRF-CBIR) plot. PRFCBIR will utilize execution get of centrality information and certification customer's interest need meanwhile. The new PRF-CBIR contains three levels: 1) private inquiry; 2) private analysis; 3 ) adjacent recuperation Private sales plays out covered solicitation with security controllable portion

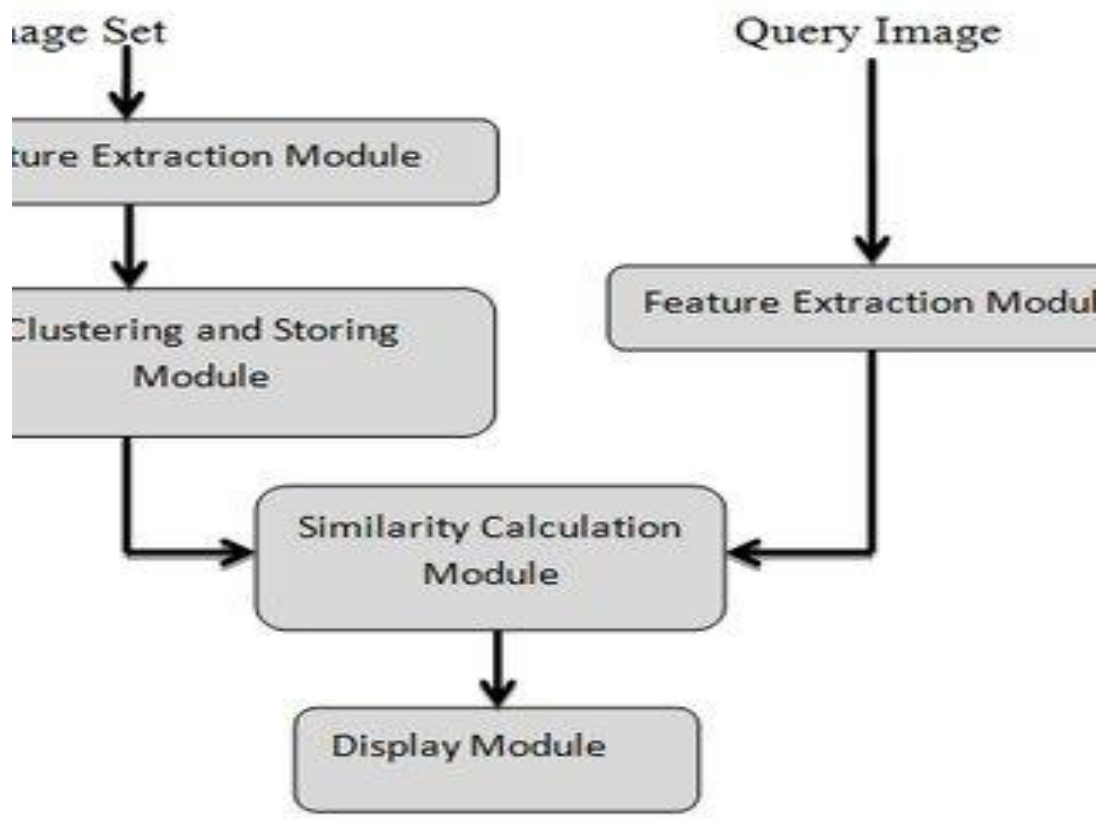

Fig. 1 System Architecture

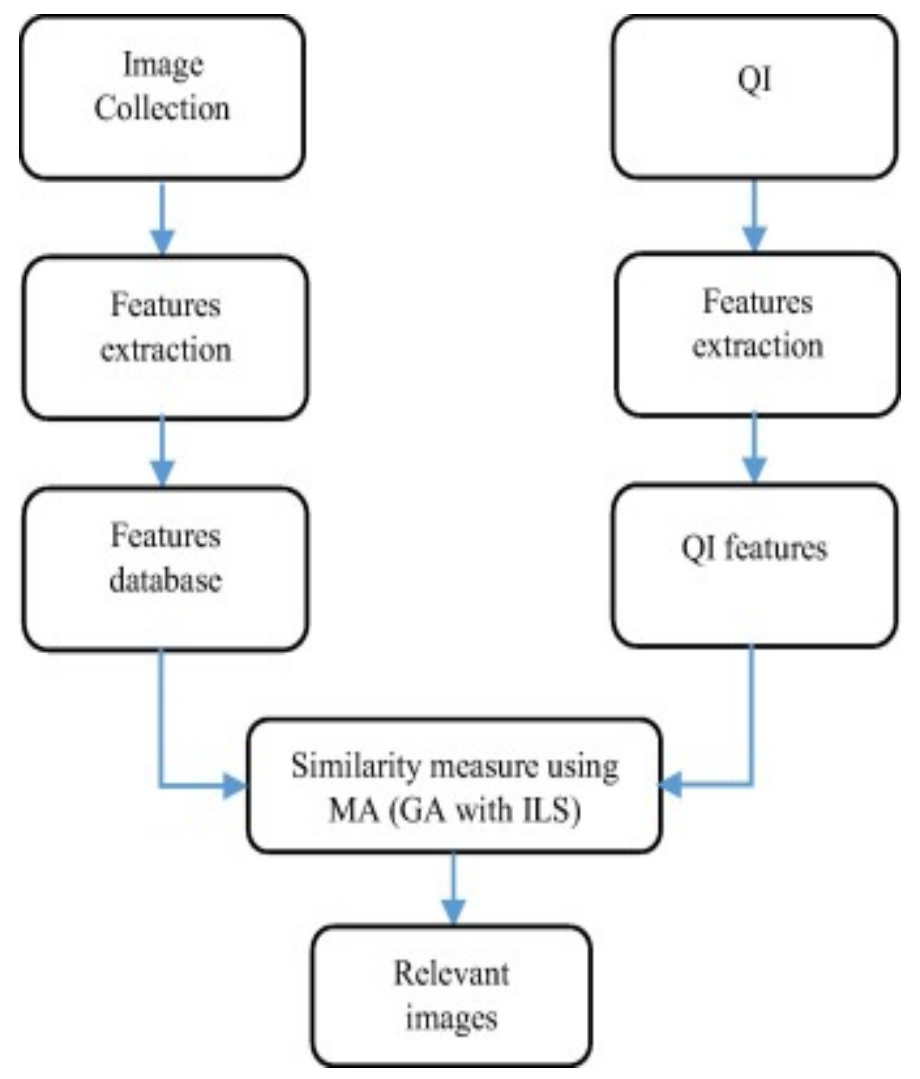

Fig. 2 System work flow vector; private information makes the data picture set by displaying astounding classes following the K-absence of clearness rule; near to recovery at long last re-positions the photographs in the client side. Security examination demonstrates that PRF-CBIR satisfies the protection necessities. The tests completed on this present reality picture gathering confirm the sensibility of the proposed PRF-CBIR plot.

\section{Architecture}

Feature Extraction Modul 


\section{Results}

The above architecture facilitate us to recognize more evidently how the data will work from stage to stage including the illustrative view of the proposal represented in the work.

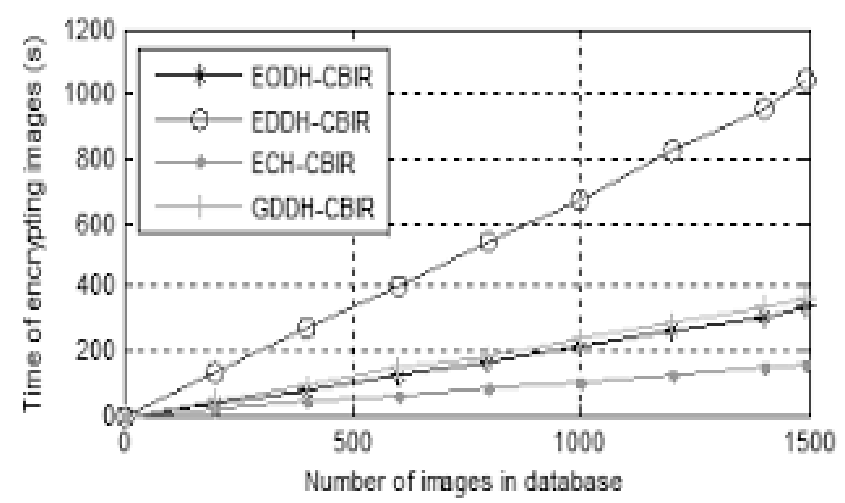

Fig. 3 No of images in database

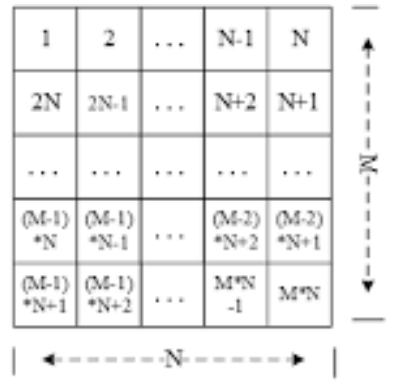

(a)

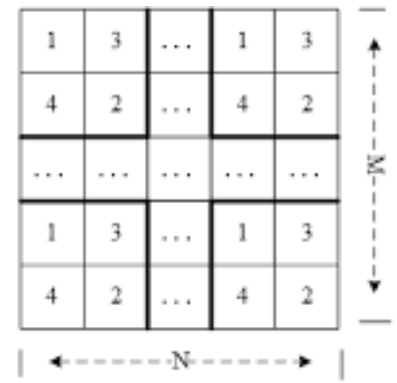

(b)

Fig. 4 graph

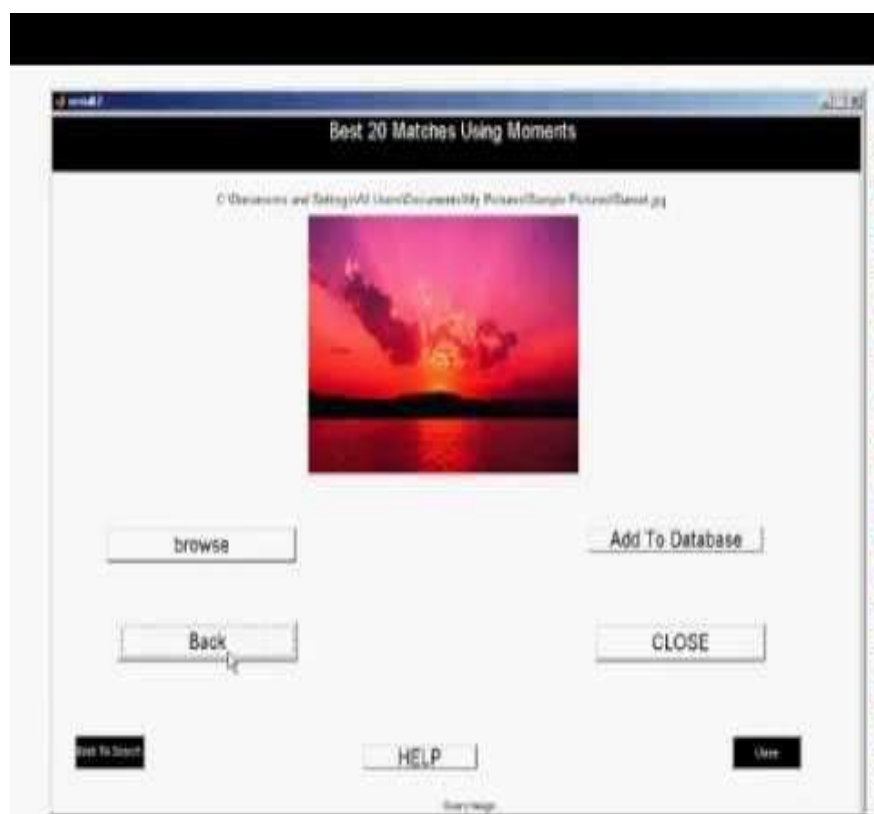

Fig. 5 Output screenshot

\section{CONCLUSION}

This paper tended to another issue of security assurance in RFCBIR. Another PRF-CBIR plan is proposed that secure the client's hunt aim as well as influence the presentation increase of pertinence input. PRF-CBIR comprises of the phase: private inquiry, private input including nearby recovery. PRF-CBIR which manages question assault, result assault and input assault existing in RF-CBIR. The system gave a hypothetical investigation over security assurance of new plan. System demonstrates new plan which adequately control security spillage and fundamentally decrease the assault achievement likelihood. In addition, we did countless tests on a true picture accumulation. The outcomes exhibit that the protection safeguarding execution of PRF-CBIR is fundamentally improved contrasted with RF-CBIR, while recovery execution penance remains satisfactory. Achievement rate of criticism assault including OFI (Optimal Frequency Inferring) decreases drastically from $100 \%$ in RF-CBIR to $41 \%$ in PRF-CBIR $(K=5)$, at the same time as normal exactness of top 20 diminishes somewhat from $46 \%$ to $42 \%$. We additionally researched the parameters of the new plan and give the proposals.

In the paper we have proposed another sheltered framework for the assurance protecting redistributed limit, look, additionally, recuperation of sweeping scale, logically revived picture documents, where abatement of client overheads remains central point of view. In the reason of proposed system is a novel cryptographic arrangement, expressly proposed for pictures, named IES-CBIR. In case of its structure the importance given to was the recognition that in pictures, concealing data can be disconnected from surface information, engaging the usage of various encryption techniques with various properties for each one as well as allowing security shielding Content-Based Image Retrieval to be performed by outcast, untrusted cloud servers. Officially research done on the security of our suggestions, as well as added preliminary appraisal of executed models depicts that our philosophy attains the interesting trade off among precision and survey in CBIR, while indicating unrivalled as well as versatility when differentiated and elective courses of action. As further work we plan for unequivocally address symmetrical issues in our plan, for example, key sharing, client get to control, and unwavering quality issues. Then, additionally map to research the importance of our technique - for instance the partition of data settings while taking care of data (concealing and surface in this paper) - in various regions previous picture data.

\section{REFERENCES}

1. Zarita Zainuddin, Ong Pauline, "Function Approximation Using Artificial Neural Networks", in International Journal of Systems Applications, Engineering \& Development, Issue 4, Volume 1,2007 2015 IEEE Conference on Systems, Process and Control (ICSPC 2015), 18 - 20 December 2015, Bandar Sunway, Malaysia 122.

2. Prateek Verma, Maheedhar Dubey, Praveen Verma and Somak Basu, "Daughman's Algorithm Method For Iris Recognition-A Biometric Approach", in International Journal of Emerging Technology and Advanced Engineering, ISSN 2250-2459, Volume 2, Issue 6, June 2012

3. Ankita Agrawal and Ashish Bansal, "Online Attendance Management System Using RFID with Object Counter", in International Journal of Information and Computation Technology. ISSN 0974-2239 Volume 3, Number 3 (2013), pp. 131-138. 
4. Pallavi Verma1 Namit Gupta2, "Fingerprint Based Student Attendance System Using GSM", in International Journal of Science and Research (IJSR) ISSN (Online): 2319-7064.

5. S. Kardy and M. Smaili, "Wireless Attendance Management System based On Iris Recognition", in Scientific Research and Essays, Vol. 5(12), 18 June 2010, pp. 1428-1435.

6. Vishal Bhalla, Tapodhan Singla, Ankit Gahlot, Vijay Gupta, "Bluetooth Based Attendance Management System", in International Journal of Innovations in Engineering and Technology (IJIET).

7. D.H. Shin J. Jung and B.H. Chang, "The psychology behind QR codes: User experience perspective," Computers in Human Behaviour, vol. 28, 2012, pp. 1417-1426

8. T. Jamil, "Automatic attendance recording system using mobile phone," Telecommunication Forum (TELFOR), 2011, pp. 1297-1299.

9. bM.M. Rahim, A.H. Seyal and M.N. Rahman, "Use of software development methods: An empirical study in Brunei Darussalam," Information and Software Technology, vol.39, no.14-15, 1998, pp. 949- 963

10. Shehu and A. Dika, "Using real time computer vision algorithms in automatic attendance management systems," in Proc. 32nd International Conference on Information Technology Interfaces (ITI), 2010, pp. 397- 402.

11. J.W. Westerman, L.A. Perez-Batres, B.S. Coffey and R.W. Pouder, "The relationship between undergraduate attendance and performance revisited: Alignment of student and instructor goals," Decision Sciences Journals of Innovative Education, vol. 9, no. 1, pp. 49-67, 2011.

12. S. Dey, S. Barman, R.K. Bhukya, R.K. Das, B.C. Haris, S.R.M. Prasanna and R. Sinha, "Speech biometric based attendance system," in Proc. Twentieth National Conference on Communications (NCC), 2014, pp. 1-6.

13. Abdulkareem, D.U. Ike and F. Olowononi, "Development of a radio frequency identification based attendance management application with a pictorial database framework," International Journal of Research in Information Technology, vol. 2, no. 4, 2014, pp. 621-628.

14. D.N. Singh and C.K. Munukoti, "Attendance monitoring system using ARM9 with QR code,", International Journal of Latest Trends in Engineering and Technology (IJLTET), vol. 2, no. 1, January 2013, pp. 246-250. 